\title{
The Price Restriction and the Distribution of Extreme Returns in the Chinese Stock Market
}

\author{
Catalina Garca*, Jose Maria Perez Sanchez
}

Quantitative methods for economics and Business, Granada University, Spain

*Corresponding Author: cbgarcia@ugr.es

Copyright (C) 2017 by authors, all rights reserved. Authors agree that this article remains permanently open access under the terms of the Creative Commons Attribution License 4.0 International License

\begin{abstract}
The distribution for the adjustment of financial returns must be capable not only to successfully completing the adjustment stage, but must also recover the stochastic characteristics of the sample data, including the bounded domain. This assumption is even more logical in the Chinese stock market where there is a price restriction in the daily movement among other special characteristics. This paper analyzes if the price restriction affects to the distribution of extreme returns by using the Peak Over Threshold (POT) method to model the extreme behavior for the daily Shanghai Stock Exchange (SSE) Composite Index applying the Generalized Pareto distribution (GPD). The risk measures calculated allow to conclude that the restriction of prices leads to a lesser risk.
\end{abstract}

Keywords Risk Management, Chinese Stock Market, Bounded Distributions, Extreme Returns

\section{Introduction}

The normality assumption presented by [1] has been displaced due to empirical evidence shows that financial returns are leptokurtic and generally skewed to the left, [2], [3], [4] and [5]. In addition to slight asymmetry and high kurtosis, the financial returns present another relevant feature: they are defined in a limit domain. [6] focused on the application of statistical mechanics to finance proposing to search for a model providing optimal description of financial markets. Thus, the distribution employed for the adjustment should recover the stochastic characteristics of the sample data. Despite of this fact, the financial returns are generally fitted with unbounded distributions. It seems logical that if the financial returns are finite, they should be fitted with a bounded distribution. This assumption could be even more logical in the case of Chinese financial returns taking into account that the short-sale trading is strictly prohibited and there is a limit of 10 percent in the daily movements in share price since 16 December 1996, [7]. Another interesting feature is that more than 80 percent of investors in Chinese stock market are individuals. All these characteristics make Chinese stock market unique to be deeply analyzed.

There are many studies on the Chinese stock market such as [8] who studied its microestructure or [9] and [10] who reviewed the pricing and the returns properties. However, the analysis of the extreme stocks returns in Chinese stock market is still emerging. [11] presented a revision about the Chinese stock market focusing on the distribution of extreme returns of Shangai Stock Exchange (SSE) index and concluding that the impact of the stock movement restriction is limited since the mean movement of this index is between $-1 \%$ and $1 \%$. On the other hand, [12] analyzed the Hang Seng index concluding that alternative bounded distributions, such as the Uniform Two sided power distribution [13] and [14], presents a better fit than the normal one. Based on this idea, it seems logical that not only the stock returns but also its tails should be fitted by a bounded distribution and this paper deals with this topic from an empirical point of view. The application of bounded distributions could also affect to the calculation of risk measures such as the Value at Risk (VaR).

Measuring the risk is an essential issue for financial institutions and investors in general and the VaR has become a basic and widespread tool for this task. Although the concept of VaR is very simple, its calculation could be rather complex. 
[15] presented a comprehensive review of value at risk methodologies placing the extreme value theory (EVT) within the semi-parametrics methodology and distinguishing two methods: the block maxima model (BMM) and the peaks-over threshold model (POT). The BMM splits the temporal horizon into blocks taking into account the maximum values in each period providing a sequence of maximum blocks that are adjusted by a generalized distribution of extreme values. This methodology, commonly used in hydrology and engineering applications, seems not be very useful for financial data since the cluster phenomenon largely observed in financial returns. Many authors mention that traditional block maxima methodology should only be applied in idyllic situations ensuring i.i.d. distributions and non-heavy tails and even [16] proposed a modified block maxima methodology. However, the paper recently published by [11] applied the BMM to model the distribution of extreme financial returns by using daily data from Shangai Stock Exchange (SSE) composite index. This paper aims to fill the gap by modelling this same data but using the POT methodology which is considered to be more useful for practical applications due to the more efficient use of the data for the extreme analysis.

The POT methodology considers the sorting of the clustered phenomena that are frequently found in data and model it based on the Generalized Pareto distribution (GPD) to describe tail behaviour, [17]. Alternatively, extreme tail returns could be modelled with semi parametric methods or related estimators (such as the Hill or Picklands estimators), although the approaches are asymptotically analogous. [18] applied the POT to investigate the tail behaviour of Asian equity market returns and quantifies two risk measures; [19] modeled VaR for long and short trading positions in oil market by applying both unconditional and conditional EVT models to forecast Value at Risk; [20] discussed tail specific distributions based on EVT and [21] estimated tail-related risk measures using extreme value theory (EVT) in the Indian stock market. All these works apply the POT to model the extreme behavior in financial markets but none consider data from Chinese stock market which, as mentioned above, presents interesting and unique characteristics that motivate this analysis.

Focused on the analysis of the long term extreme Chinese returns behaviour, this paper applies the extreme value theory using the unconditional Peaks over Threshold method to generate the risk measures with tail returns modelled with a heavy-tailed Generalised Pareto Distribution (GPD). Depending on the estimation of the parameter $\xi$, this distribution can present a bounded $(\xi<0)$ or unbounded domain $(\xi>0)$. [22] applied the GPD distribution to quantify the market risk by the tail distribution estimation of six market indices (ES50, FTSE100, HS, Nikkei, SMI and S\&P500) obtaining a point estimation of $\xi$ always greater than zero. We use data from daily financial returns of Shangai Stock Exchange (SSE) previously applied by [11]. The aim of the paper is to analyze if the price restriction influences on the distribution of the extreme returns leading to the bounded version of the GPD distribution $(\xi<0)$. We also analyze if the restriction price has any influence on the risk measures.

This paper is organized as follows. In methodology section we revise the peak over threshold methodology explaining the process to select the threshold $u$ and remembering the GPD distribution and its maximum likelihood estimation. In the empirical section we apply the described methodology to daily log return for Shangai Stock Exchange (SSE) composite index for the full period from 2 January 1991 to 31 December 2013 and for the period from 2 January 1991 to 16 December 1996 when the restriction price were not implemented. We obtain the maximum likelihood estimation of the GPD and risk measures for both periods and compare results. The last section presents the main conclusions.

\section{Methodology}

Within the different methodologies to estimate the VaR, the extreme value theory focuses on the limiting distribution of extreme returns observed over a long time. There are two different methodologies: the block maxima model (BMM) that considers the maximum values that the variable takes in successive periods and the peak-over-threshold (POT) approach focuses only on the observations exceeding a given (high) threshold. Based on its empirical superiority and being interesting in long term behavior, in this paper we adopt the unconditional POT model to identify the extreme observations that exceed a high threshold $u$. Among others, [22] suggested that the $u$ should be high but keeping an equilibrium since the higher the threshold the less observations are present in the estimation of the tail distribution function. Although several techniques are available for threshold determination, there is no methodology to automatically select the threshold $u$.

From a sample ordered in a descendent way, it is practical to use the data of the sample as initial threshold, [23], it is to say that $u=X_{k+1}$, and then the empirical function of the excess mean is defined as the sum of the excesses over the threshold $u$ divided by the number of data points which exceed the threshold $u$ and is expressed as:

$$
e_{n}(u)=\frac{\sum_{i=1}^{k} X_{i}}{k}-X_{k+1}, \quad k=1, \ldots, n-1
$$


where $k$ is the number of observations exceeding the threshold $u$. The scientific literature coincides that the "sample mean excess plot" is a very helpful graphical tool defined by the points $\left(u, e_{n}(u)\right)$. The sample mean excess function should be linear and this property is used as a criterion for the selection of $u$.

After selecting the threshold, the problem will be to estimate the distribution function $F_{u}$ of $x$ above a certain threshold $u$. The distribution function $F_{u}$ is called the "conditional excess distribution function" and is defined as:

$$
F_{u}(y)=P(X-u \leq y \backslash X>u), \quad 0 \leq y \leq x_{F}-u,
$$

where $X$ is a random variable, $u$ is a given threshold, $y=x-u$ are the excesses and $x_{F} \leq \infty$ is the right endpoint of $F$. Then, $F_{u}(y)$ can be written as:

$$
F_{u}(y)=\frac{F(u+y)-F(u)}{1-F(u)} \frac{F(x)-F(u)}{1-F(u)} .
$$

It is possible to obtain different risk measures assuming a concrete distribution function for the tail distribution $F_{u}(y)$. [24], [25] stated that the conditional excess distribution function $F_{u}(y)$ is well approximated by the Generalized Pareto distribution (GPD) that is defined with the following cumulative distribution function:

$$
H_{\xi}(y)= \begin{cases}1-\left(1+\frac{\xi}{\sigma} y\right)^{\frac{-1}{\xi}}, & \text { if } \xi \neq 0 \\ 1-e^{\frac{-y}{\sigma}}, & \text { if } \xi=0\end{cases}
$$

where $0<y<\left(x_{F}-u\right)$ if $\xi>0$ and $0 \leq y \leq \frac{-\sigma}{\xi}$ if $\xi<0$ being the bounded version of the GPD distribution. Indeed, a negative $\xi$ is associated with very thin-tailed distributions while a value of $\xi>0$ is associated with heavydistributions more common in financial returns. This paper applies this distribution to analyze if the price restriction of Chinese stock market influences over the distribution of the extreme returns leading to the bounded version of the GPD distribution, $\xi<0$.

Different methods can be used to estimate the parameters of the Generalized Pareto distribution (GPD). See [26] for comparisons and detailed discussion. We will use the maximum likelihood estimation.

Given a sample $y_{1}, y_{2}, \ldots, y_{n}$ the log-likelihood function $L(\xi, \sigma \mid y)$ for the GPD is given by the logarithm of the joint density of the $n$ observations:

$$
L(\xi, \sigma \mid y)= \begin{cases}-n \log \sigma-\left(\frac{1}{\xi}+1\right) \sum_{i=1}^{n} \log \left(1+\frac{\xi}{\sigma} y_{i}\right), & \text { if } \xi \neq 0 \\ -n \log \sigma-\frac{1}{\sigma} \sum_{i=1}^{n} y_{i}, & \text { if } \xi=0 .\end{cases}
$$

\subsection{Risk measures}

The Value at Risk (VaR) is considered the most popular measure used in the financial risk management area and it is defined as a quantile measure that represents the maximum loss for a given probability $(p)$. However, this measure founded important criticism since it does not take into account the possible losses exceeding VaR. Contrarily to the VaR, the Excess Shortfall (ES) takes account of the magnitude of losses exceeding the VaR being always bigger than the VaR. Indeed, the ES is defined as the average loss if the VaR quantile $(p)$ is exceeded. Both measures are directly obtained from the distribution of excesses. Firstly, we will have to isolate from expression (3)

$$
F(x)=(1-F(u)) F_{u}(y)+F(u),
$$

then, we replace $F_{u}(y)$ by the selected distribution function and $F(u)$ by the estimate $\frac{n-N_{u}}{n}$, where $n$ is the total number of observations and $N_{u}$ the number of observations above the threshold $u$.

Assuming a GPD function for the tail distribution, it is possible to obtain the following expressions for the $\mathrm{VaR}_{p}$ and the $\hat{E S}$, respectively:

$$
\begin{gathered}
\hat{\operatorname{VaR}}_{p}=u+\frac{\hat{\sigma}}{\xi}\left(\left(\frac{n}{N_{u}} p\right)^{-\xi}-1\right), \\
\hat{\mathrm{ES}_{p}}=\hat{\mathrm{VaR}}_{p}+E\left(X-\hat{\operatorname{VaR}}_{p} \mid X>\hat{\operatorname{VaR}}_{p}\right) .
\end{gathered}
$$

These measures are tailed dependent so we calculate them separately for right and left tails representing long and short trading positions, respectively $([\overline{22}])$. 


\section{Empirical Application}

\subsection{Data}

Although the Chinese stock market is the second largest stock market behind US stock market, it has some relevant peculiarities that difference this market from developed stock market. Firstly, the most of investors are individual contrarily to developed markets and, therefore, the budget may be more restricted. In addition, the short-sale trading is strictly prohibited and there is a limit of 10 percent in the daily movement in share pricing since 16 December 1996, [7]. Another difference is that there are different kinds of shares: A shares restricted to domestic investors and B shares available to both domestic and foreign investors. In addition, $\mathrm{H}$ shares are traded on the Hong Kong Stock Exchange in Hong Kong dollars but regulated by Chinese law. All these features make the Chinese stock market unique and interesting.

We use daily data of Shangai Stock Exchange (SSE) Composite Index that is a stock market index of all stocks (A and B shares) that are traded at the largest stock market in continental China, the Shanghai Stock Exchange. Data were obtained from Yahoo Finance from 2 January 1991 to 31 December 2013. Following [11], who previously applied these data, the return of 21 May 1992 is treated as an outlier and is removed from the sample. Returns are obtained as $R_{t}=\ln \frac{p_{t}}{p_{t-1}}$. Figure 1 presents the daily log returns where we can note the price regulation from December 1996.

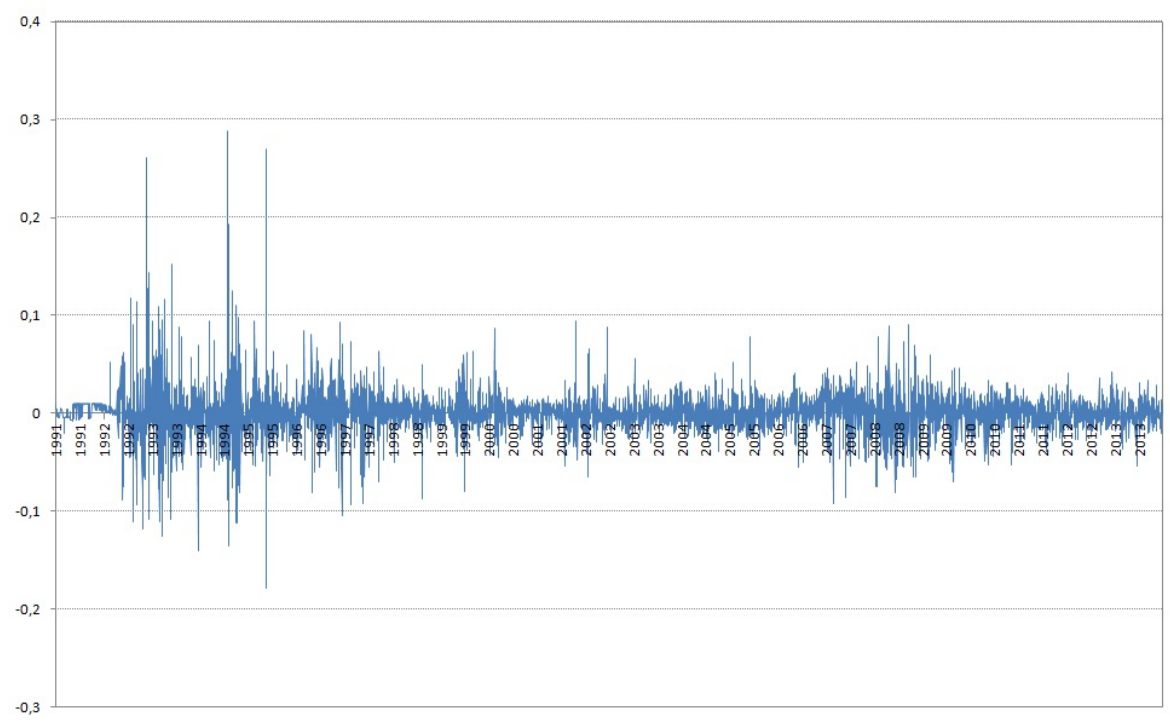

Figure 1. Daily log returns for Shangai Stock Exchange (SSE) Composite Index obtained from 2 January 1991 to 31 December 2013.

Table 1. Main statistical characteristics of daily returns of Shangai Stock Exchange (SSE) Composite Index

\begin{tabular}{ccc}
\hline Period & 2-jan-1991 to 31-dec-2013 & 2 jan-1991 to 16-dec-1996 \\
\hline N & Full period & Not Pricing restriction period \\
\hline Minimum & 5903 & 1542 \\
Maximum & -0.179051 & -0.179051 \\
Mean & 0.288610 & 0.288610 \\
Standard deviation & 0.000352 & 0.00086311 \\
CV & 0.021821 & 0.032953 \\
Skewness & 0.016144 & 0.0261918 \\
Kurtosis & 1.207644 & 1.39633 \\
Jarque-Bera $(p$-value $)$ & $6.06726 e+006(0.0000)$ & $553048(0.0000)$ \\
\hline
\end{tabular}


Table 1 shows the main statistical returns of daily returns of Shangai Stock Exchange (SSE) Composite for the full period from 2 January 1991 to 31 December 2013 and from the period where the price was not restricted from 2 January 1991 to 16 December 1996 . Note that the maximum and minimum vales are -0.179051 and 28.288610 and coincide for both periods so the extreme values come from the period were the restriction price was not in force. The unconditional mean of daily log-returns is close to zero in both periods but the coefficient of variation is lower for the full period denoting, as expecting, that the data with price restriction are more homogenous. Note that in both periods there is a positive asymmetry and a high kurtosis rejecting the assumption of normality as concluding by the Jarque-Bera test.

We consider both the left and the right tails of the return distribution since represent losses for investors with long and short position on the index, respectively. The implementation of the POT starts with the selection of the threshold $u$ to fit the GPD function for the excess over $u$ and then estimate the parameters of the GPD distribution to finally obtain the estimation of the risk measures.

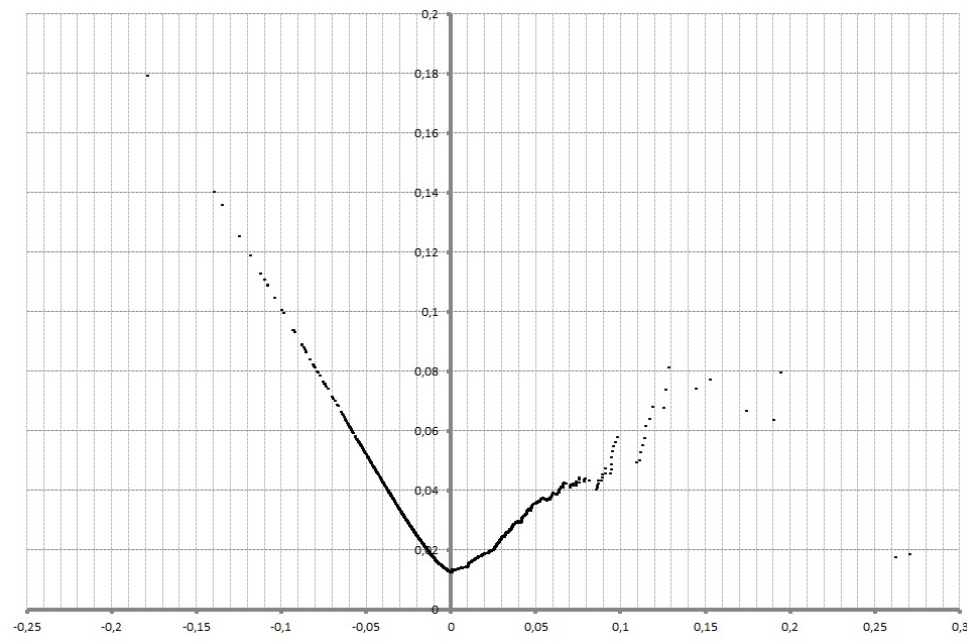

(a) Sample mean excess plot for the right tail

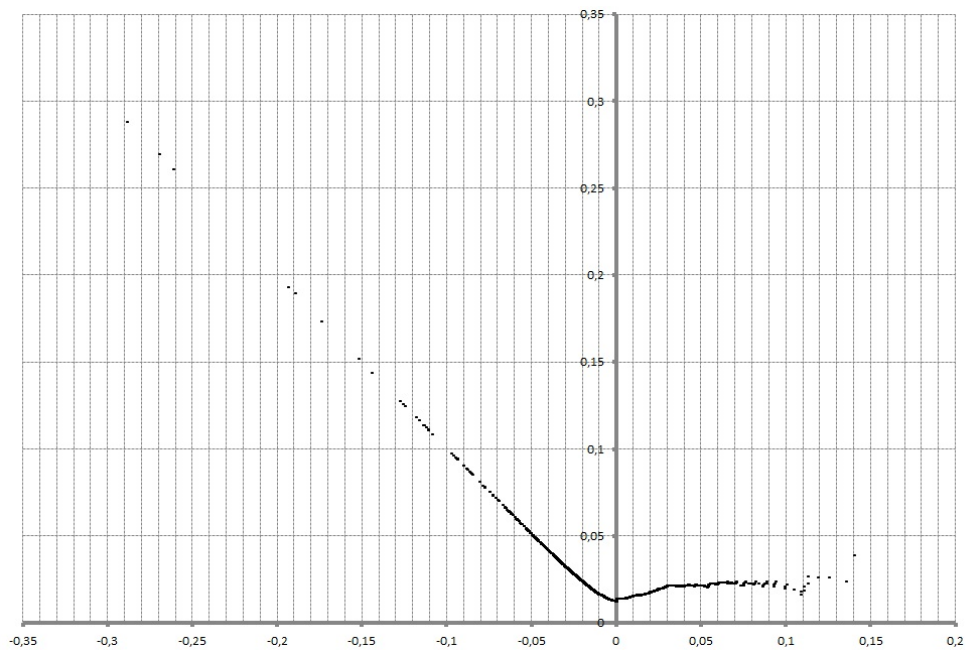

(b) Sample mean excess plot for the left tail.

Figure 2. Determination of the threshold $u$

\subsection{Selection of threshold}

As mentioned above, the POT method using the GPD is applied for tail estimation of the standardized residual series. The first step in this modeling is to estimate the threshold for identifying the relevant tail region. Figure 2 displays the sample mean excess plot corresponding to the SSE data. While the representation of the excess mean plot of the right tail was obtained directly applying the expression (1), the signs of the return were changed in the left tail case so that positive 
values correspond to losses. A closer inspection of the plots suggests the values $u=0.01$ for the threshold of the right tail and $u=0.005$ for the threshold of the left tail. These values leave 1256 and 1835 observations in the tails, respectively.

Table 2 reports the descriptive statistics for the right and the left tails. The mean is around zero in both tails and the coefficient of variation indicates that both tails present similar dispersion. The skewness and kurtosis suggest the nonnormality of the distribution that is confirmed by the Jarque-Bera test. The skewness and kurtosis are greater for the right tail than for the left one indicating that the upper tail is likely fatter than the lower one.

Table 2. Main statistical characteristics of right and left tail for the period 2 January 1991 to 31 December 2013

\begin{tabular}{ccc}
\hline Tail & Right & Left \\
\hline $\mathrm{N}$ & 1256 & 1835 \\
Minimum & 0.01 & 0.005 \\
Maximum & 0.26992 & 0.14014 \\
Mean & 0.02529 & 0.01937 \\
Standard Deviation & 0.02198 & 0.017138 \\
CV & 1.15059 & 1.13023 \\
Skewness & 4.55929 & 2.604819889 \\
Kurtosis & 32.98087 & 9.00821 \\
Jarque-Bera $(p$-value) & $60797.8(0.00000)$ & $8237.92(0.00000)$ \\
\hline
\end{tabular}

On the other hand, Table 3 reports the descriptive statistics for the right and the left tails for the period without price restriction from 2 January 1991 to 16 December 1996. We have kept the same threshold selected previously since the "sample mean excess plot?' is very similar. Then, the threshold is $u=0.01$ for the right tail and $u=0.005$ for the left tail leaving 358 and 525 observations respectively. The mean is around zero for both tails although the coefficient of variation is lesser in the right one concluding that these data are more homogenous. Both tails show right asymmetry and, in relation to kurtosis the right tail presents a higher kurtosis although in both tails the normality assumption is rejecting by using the Jarque-Bera test.

Table 3. Main statistical characteristics of right and left tail for period from 2 January 1991 to 16 December 1996

\begin{tabular}{ccc}
\hline Tail & Right & Left \\
\hline $\mathrm{N}$ & 358 & 525 \\
Minimum & 0.01 & 0.005 \\
Maximum & 0.288610 & 0.140140 \\
Mean & 0.037374 & 0.0261017 \\
Standard Deviation & 0.036641 & 0.02330 \\
CV & 0.818671 & 1.120168 \\
Skewness & 3.37238 & 1.957605 \\
Kurtosis & 15.72688 & 4.345131 \\
Jarque-Bera $(p$-value $)$ & $4252.65(0.00000)$ & $736.455(1.20475 \mathrm{e}-160)$ \\
\hline
\end{tabular}

\subsection{Maximum likelihood estimation}

Table 4 shows the values $\hat{\xi}$ and $\hat{\sigma}$ that maximize the log-likelihood function $(\hat{f})$ for the sample defined by the observations exceeding the threshold $u$ in the period from 2 January 1991 to 31 December 2013. We obtain the estimates $\hat{\xi}=-0.0440755$ and $\hat{\sigma}=0.0263519$ for the right tail and $\hat{\xi}=-0.0737752$ and $\hat{\sigma}=0.0207619$ for the left tail. Figure 3 displays the relative log-likelihood functions for both tails. 
Table 4. Parameter estimates for period from 2 January 1991 to 31 December 2013

\begin{tabular}{cccccc}
\hline Tail & $u$ & $N_{u}$ & $\hat{\xi}$ & $\hat{\sigma}$ & $\hat{f}$ \\
\hline Right & 0.01 & 1256 & -0.0440755 & 0.0263519 & 3366.44 \\
Left & 0.005 & 1835 & -0.0737752 & 0.0207619 & 5410.34 \\
\hline
\end{tabular}

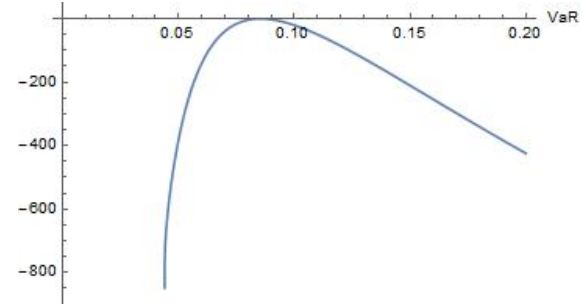

(a) Right tail

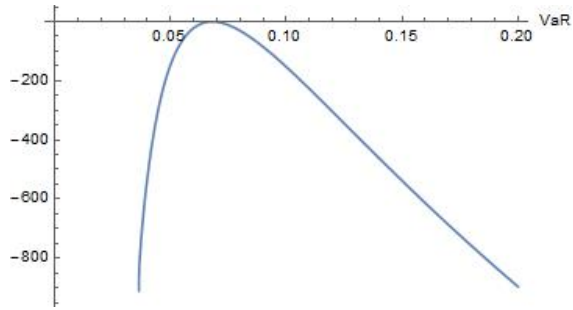

(b) Left tail.

Figure 3. Relative profile log-likelihood function with price restriction.

In table 5 we show the results by repeating the process for the data of the period from 2 January 1991 to 16 December 1996, when the price restriction was not implemented, obtaining the estimates $\hat{\xi}=-0.0108538$ and $\hat{\sigma}=0.037$ for the right tail and $\hat{\xi}=-0.097602$ and $\hat{\sigma}=0.0286191$ for the left tail. Figure 4 displays the relative log-likelihood functions for both tails.

Table 5. Parameter estimates for period from 2 January 1991 to 16 December 1996

\begin{tabular}{cccccc}
\hline Tail & $u$ & $N_{u}$ & $\hat{\xi}$ & $\hat{\sigma}$ & $f$ \\
\hline Right & 0.01 & 358 & -0.0108538 & 0.037 & 818.706 \\
Left & 0.005 & 525 & -0.097602 & 0.0286191 & 1391.92 \\
\hline
\end{tabular}

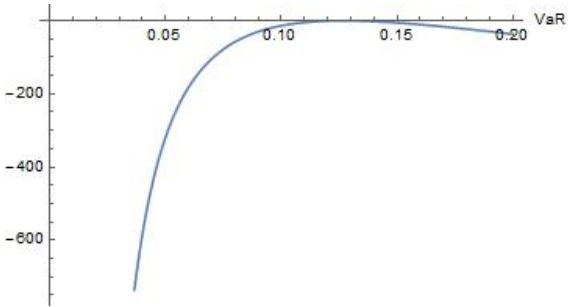

(a) Right tail

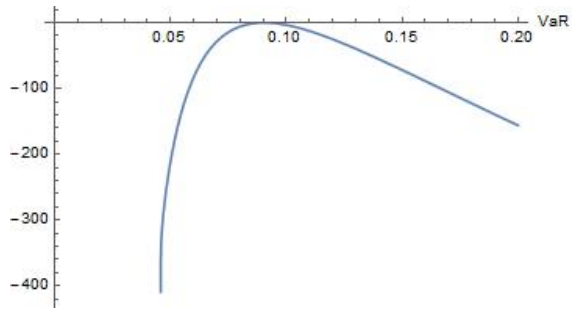

(b) Left tail.

Figure 4. Relative profile log-likelihood function without price restriction.

Note that for both periods the estimation of the parameter $\xi$ becomes $<0$ leading to the bounded version of the GPD distribution. This suggests that the influence of the price restriction over the estimation of the parameter is limited but it is still recommended to use a bounded distribution. This can be motivated by other special features of Chinese stock market such as the great percentage of individual investors in comparison with other development stock markets.

\subsection{Obtention of risk measures}

The VaR and the ES can be directly computed from expressions (7) and (8) respectively. Table 6 collects the values of the Var and the ES for the right and the left tails for $p=0.01$. Note that the left tail has a lower Var and Es than the right one. 
Table 6. Main risk measures for full period (2 January 1991 to 31 December 2013) and the not price restricted period (2 January 1991 to 16 December 1996)

\begin{tabular}{cccc}
\hline Tail & Period & $\hat{\operatorname{Var}}_{p}$ & $\hat{\mathrm{ES}_{p}}$ \\
\hline Right tail & 2 January 1991-31 December 2013 & 0.085375 & 0.107431 \\
Right tail & 2 January 1991-16 December 1996 & 0.126801 & 0.162919 \\
Left tail & 2 January 1991-31 December 2013 & 0.0680261 & 0.0830312 \\
Left tail & 2 January 1991-16 December 1996 & 0.0904141 & 0.108893 \\
\hline
\end{tabular}

Comparing the right and left tails, we observe that the right tail presents always a $\hat{V a R}_{p}$ and $\hat{E S}_{p}$ greater to the ones obtained to the left tail. Thus, regardless to the price restriction the right tail presents more risk than the left one. On the other hand, note that the VaR and the ES obtain higher estimations in the period when the price restriction was not implemented and then we can conclude, as was expected, that the risk is lesser when there exists a price restriction.

\section{Conclusions}

This paper examines the extreme risk in Chinese stock market and analyzes the influence of the price restriction implemented from 16 December 1996 on the distribution of the extreme returns and risk measures. Being interesting in the long term, it is applied the unconditional Peak Over Threshold (POT) with the Generalized Pareto distribution (GPD). This distribution can present a bounded or unbounded domain depending on the estimation of the parameter $\xi$. Our first conclusion is that, contrarily to what happens with other indices, [22], the Shangai Stock Exchange (SSE) composite index leads to the bounded version of the GPD $(\xi<0)$ when we estimate it by maximum likelihood. On the other hand, we compare this result with the result obtained for not price restricted period concluding that also the estimation leads to the bounded version. This suggests that the influence of the price restriction is limited but also that other special characteristics of Chinese stock market, such as the great number of individual investors with limited budget, could recommend to work with a bounded domain.

We also present two risk measures: the Value at Risk (VaR) that measures the loss up to a certain predefined threshold and the Excess Shortfall (ES) that measures the mean of the losses beyond the quantile. We present the estimation of these risk measures for the full period from 2 January 1991 to 31 December 2013 and for the period from 2 January 1991 to 16 December 1996 when the restriction price was not implemented. We obtain that both, the VaR and the ES, are lesser when the restriction price is established and consequently the risk is reduced as was pretending with the implementation of this limitation. The results are based on the analysis of daily returns and it may have different and meaningful variations from long term viewpoint with weekly or monthly returns strategy.

As a future research line we propose to analyze the application of other alternative bounded distributions and to estimate the VaR reflecting the current volatility background by using conditional extreme value theory.

\section{REFERENCES}

[1] Eugene F Fama. The behavior of stock-market prices. Journal of business, pages 34-105, 1965.

[2] François M Longin. The asymptotic distribution of extreme stock market returns. Journal of business, pages 383408, 1996.

[3] Eric Jondeau and Michael Rockinger. Testing for differences in the tails of stock-market returns. Journal of Empirical Finance, 10(5):559-581, 2003.

[4] Konstantinos Tolikas and Gareth D Gettinby. Modelling the distribution of the extreme share returns in singapore. Journal of Empirical Finance, 16(2):254-263, 2009.

[5] María del Mar López Martín, Catalina García García, and José García Pérez. Treatment of kurtosis in financial markets. Physica A: Statistical Mechanics and its Applications, 391(5):2032-2045, 2012.

[6] Rosario N Mantegna, Zoltán Palágyi, and H Eugene Stanley. Applications of statistical mechanics to finance. Physica A: Statistical Mechanics and its Applications, 274(1):216-221, 1999. 
[7] Dongwei Su and Belton M Fleisher. Risk, return and regulation in chinese stock markets. Journal of Economics and Business, 50(3):239-256, 1998.

[8] Cheng Kenneth Xu. The microstructure of the chinese stock market. China Economic Review, 11(1):79-97, 2000.

[9] Jie Zhu. Testing for expected return and market price of risk in chinese ab share market: a geometric brownian motion and multivariate garch model approach. CREATES research paper, (2008-15), 2008.

[10] Cornelis A Los and Bing Yu. Persistence characteristics of the chinese stock markets. International Review of Financial Analysis, 17(1):64-82, 2008.

[11] Saiful Izzuan Hussain and Steven Li. Modeling the distribution of extreme returns in the chinese stock market. Journal of International Financial Markets, Institutions and Money, 34:263-276, 2015.

[12] Francisco Aranda, Catalina García, and J.M. Pérez. Alternative bounded distributions to model chinese financial returns. Current topics on risk analysis. ICRA and RISK 2015 conferences, pages 65-70, 2015.

[13] J.R. Van Dorp and S. Kotz. A novel extension of the triangular distribution and its parameter estimation. The Statistician, 551(1):63-79, 2002.

[14] C. García, J. García, and J.R. van Dorp. Modelling heavy-tailed, skewed and peaked uncertainty phenomena with bounded support. Statistical, methods and applications, pages 463-486, 2011.

[15] Pilar Abad, Sonia Benito, and Carmen López. A comprehensive review of value at risk methodologies. The Spanish Review of Financial Economics, 12(1):15-32, 2014.

[16] A. Cabaña and I. Serra. An approach to solve the weakness of block maxima method. Current topics on risk analysis. ICRA and RISK 2015 conferences, pages 179-186, 2015.

[17] Paul Embrechts, Sidney I Resnick, and Gennady Samorodnitsky. Extreme value theory as a risk management tool. North American Actuarial Journal, 3(2):30-41, 1999.

[18] John Cotter. Extreme risk in asian equity markets. Available at SSRN 999564, 2007.

[19] Velayoudoum Marimoutou, Bechir Raggad, and Abdelwahed Trabelsi. Extreme value theory and value at risk: application to oil market. Energy Economics, 31(4):519-530, 2009.

[20] David E Allen, Abhay K Singh, and Robert J Powell. Evt and tail-risk modelling: Evidence from market indices and volatility series. The North American Journal of Economics and Finance, 26:355-369, 2013.

[21] Madhusudan Karmakar. Estimation of tail-related risk measures in the indian stock market: An extreme value approach. Review of Financial Economics, 22(3):79-85, 2013.

[22] Manfred Gilli and Evis Kellezi. An application of extreme value theory for measuring financial risk. Computational Economics, 27(2-3):207-228, 2006.

[23] Almudena García Pérez. La teoría del valor extremo: una aplicación al sector asegurador. (10):27-54, 2004.

[24] James Pickands. Statistical inference using extreme order statistics. the Annals of Statistics, pages 119-131, 1975.

[25] August A Balkema and Laurens De Haan. Residual life time at great age. The Annals of probability, pages 792-804, 1974.

[26] Enrique Castillo and Ali S Hadi. Fitting the generalized pareto distribution to data. Journal of the American Statistical Association, 92(440):1609-1620, 1997. 